\title{
Structural Determinants for the Interactions of Chemically Modified Nucleic Acids with the Stabilin-2 Clearance Receptor
}

\author{
Hans Gaus, ${ }^{\dagger}$ Colton M. Miller, ${ }^{\ddagger}$ Punit P. Seth, ${ }^{* \dagger}$ and Edward N. Harris $*$ \\ ${ }^{\dagger}$ Department of Medicinal Chemistry, Ionis Pharmaceuticals, Carlsbad, California 92010, United States \\ ${ }^{\ddagger}$ Department of Biochemistry, University of Nebraska, Lincoln, Nebraska 68588, United States
}

Supporting Information

ABSTRACT: The Stabilin receptors are systemic clearance receptors for some classes of chemically modified nucleic acid therapeutics. In this study, the recombinant human secreted ecto-domain of the small isoform of Stabilin-2 (s190) was purified from cell culture and evaluated for direct binding with a multitude of antisense oligonucleotides (ASOs) using a fluorescence polarizationbased assay. The tested ASOs varied in their backbone composition, modification of the ribose $2^{\prime}$ position, overall length of the oligo, and sequence of the nucleotide bases. A fully phosphorothioate (PS) ASO with a 5-10-5 pattern of flanking 2'-O-methoxyethyl modifications was then used to test the effects of $\mathrm{pH}$ and salt concentration on receptor binding. These tests concluded that the PS backbone was the primary determinant for ASO binding and that decreasing $\mathrm{pH}$ and increasing salt generally increased the rate of ligand dissociation and fit within the biological parameters expected of a constitutive recycling receptor. These results will be useful in the rational design of therapeutic oligonucleotides for enhancing their affinity or avoidance of the Stabilin receptors.

A ntisense oligonucleotides (ASOs) are short (14-25) 1 chemically modified nucleic acids that have made rapid progress for the treatment of congenital and acquired metabolic diseases. $^{1}$ The effectiveness of an ASO relies on several parameters, including biological stability, adherence to cellsurface proteins, internalization within the cells, and escape from endosomes and specificity to the target RNA. ${ }^{2,3}$ To increase their stability in biological fluids, they are often designed with a phosphorothioate linkage in which the free nonbridging oxygen atom of the phosphodiester backbone is replaced with a sulfur atom, rendering the polymer resistant to nucleases. ${ }^{4}$ The PS backbone also enhances the avidity of ASO for plasma and cell-surface proteins that promote distribution to tissues and cellular accumulation. ${ }^{5}$ Gen 2 ASOs typically have the gapmer design in which a central region of DNA nucleotides is flanked by 2 -modified nucleotide analogues that further enhance nuclease stability and RNA binding affinity. ${ }^{6}$ Commonly used 2'-modified analogues used in gapmers include 2'-methoxyethyl RNA (MOE), constrained ethyl BNA (cEt), and locked nucleic acid (LNA) ${ }^{7}$ (Figure 1).

Our collaborative group discovered that the Stabilin class of receptors, of which there are two members, is responsible for the systemic clearance of phosphorothioate antisense oligonu-

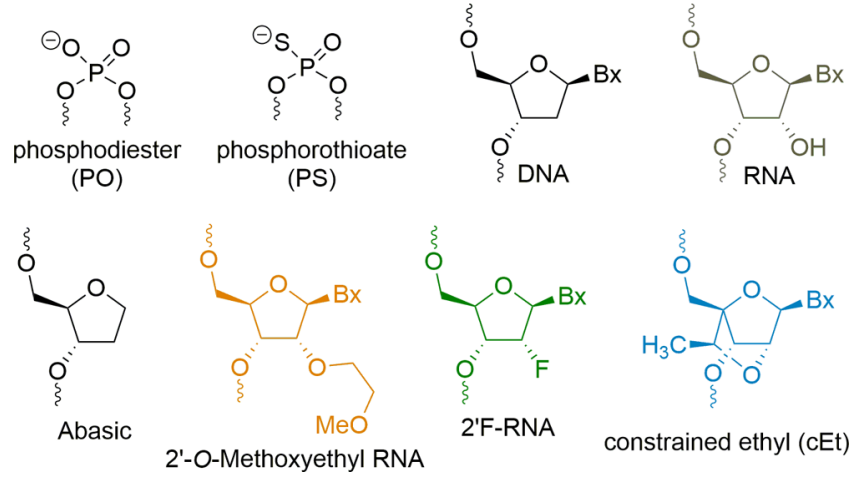

Figure 1. Structures of chemical modifications used in this study.

cleotides (PS-ASOs). ${ }^{8}$ Both human Stabilin-1 and Stabilin-2 are $\sim 315 \mathrm{kDa}$ type 1 receptors with a single transmembrane domain and a short cytoplasmic tail. ${ }^{9}$ Stabilin- 1 is more widely expressed within endothelial cells and alternatively activated macrophages. ${ }^{10}$ Stabilin-2 is expressed at a high level in the liver, spleen, bone marrow, and lymph node sinusoidal endothelium and at a lower level in specific tissues within the muscle, brain, and kidney. ${ }^{11-13}$ Both receptors share the same domain organization in which the extracellular portion consists of seven Fasciclin-1 domains separated by four clusters consisting of four to six EGF/EGF-like domains, and an XLink domain that binds hyaluronan in Stabilin-2 but is dysfunctional in Stabilin-1. ${ }^{14}$ Both receptors bind with ligands such as heparin, ${ }^{15}$ PS-ASOs, ${ }^{8}$ phosphatidylserine, ${ }^{16,17}$ and oxidized low-density lipoprotein. ${ }^{18}$ Each protein can also internalize their own unique ligands such as SPARC $^{19}$ and placental lactogen $^{20}$ for Stabilin-1 and hyaluronan ${ }^{21}$ and chondroitin sulfates A, C, and D for Stabilin-2. ${ }^{22}$ Sodium dodecyl sulfate-polyacrylamide gel electrophoresis analysis of the receptor demonstrates that Stabilin-1 is expressed as two high-molecular weight proteins (1:1 ratio) that migrate as a tight doublet in contrast to Stabilin-2, which is expressed as 315 and $190 \mathrm{kDa}$ isoforms in an approximately $1: 1$ ratio in native tissues. $^{23}$ For the experiments outlined in this report, we utilized the ecto-domain of the recombinant $190 \mathrm{kDa}$ isoform (s190) of Stabilin-2 as it has a high level of expression and/or secretion in cell lines and may be purified to near $100 \%$ purity

Received: February 1, 2018

Revised: $\quad$ March 22, 2018

Published: March 28, 2018 
using affinity chromatography. Both isoforms have the same activity against PS-ASOs. ${ }^{8}$

Previously, we used the recombinant $190 \mathrm{kDa}$ isoform expressed in cell lines and the s190 purified protein to assess PS-ASO binding and internalization. From both enzyme-linked immunosorbent assay (ELISA)-like assays and internalization data with $\left[{ }^{125} \mathrm{I}\right]$ PS-ASO (5-10-5 oligo), we determined that the binding affinity was $\sim 140 \mathrm{nM}^{8}{ }^{8}$ Competition assays were utilized to determine the effect of chemical modifications and oligonucleotide composition on Stab2 binding. The competition assays did not accurately inform the direct binding of the competitors or their lower affinity for the receptor. The objective in this report was to assess direct binding of a variety of ASOs to determine which chemistries (Figure 1) provide the weaker and stronger interaction between the nucleic acid and s190 using a sensitive fluorescence polarization (FP) assay. ${ }^{24,25}$

A series of experiments were performed using different variants of an ASO targeting phosphatase and tensin homologue (PTEN) mRNA to determine their affinity for s190. The interaction between the protein receptor and PTEN ASO was then challenged by $\mathrm{pH}$ and salt dependence.

Table 1 (Figure 2A) provides a summary of results for the initial binding experiments with the PTEN ASOs. It was found

\section{Table 1. Binding of PTEN ASOs to s190 (Stab2) ${ }^{a}$}

\begin{tabular}{|c|l|l|l|}
\hline ASO & Sequence & ASo Design & Kd (nM) \\
\hline $\mathbf{1}$ & X-CTGCTAGCCTCTGGATTTGA & 5-10-5 MOE PS & $12.6(18.8)$ \\
\hline $\mathbf{2}$ & X-CTGCTAGCCTCTGGATTTGA & 5-10-5 MOE MBB & 35.5 \\
\hline $\mathbf{3}$ & X-CTGCTAGCCTCTGGATTTGA & uniform DNA PS & 11.4 \\
\hline $\mathbf{4}$ & X-CTGCTAGCCTCTGGATTTGA & uniform MOE PS & 7.0 \\
\hline $\mathbf{5}$ & X-CTGCTAGCCTCTGGATTTGA & 5-10-5 MOE PO & $>1,000$ \\
\hline $\mathbf{6}$ & X-CTGCTAGCCTCTGGA & 15-mer PS & 83.9 \\
\hline $\mathbf{7}$ & X-CTGCTAGCCT & 10mer PS & $>1,000$ \\
\hline $\mathbf{8}$ & $\begin{array}{l}\text { X-CTGCTAGCCTCTGGATTTGA } \\
\text { TCAAATCCAGAGGCTAGCAG }\end{array}$ & $\begin{array}{l}\text { R-10-5 MOE PS / } \\
\text { RNAmplement }\end{array}$ & $>1,000$ \\
\hline $\mathbf{9}$ & X-CTGCTAGCCTCTGGATTTGA & 5-10-5 cEt PS & 16.1 \\
\hline $\mathbf{1 0}$ & X-CTGCTAGCCTCTGGATTTGA & 5-10-5 2'-F PS & 10.6 \\
\hline & \multicolumn{2}{|l}{} \\
\hline
\end{tabular}

${ }^{a}$ Orange letters indicate MOE, gray letters RNA, black letters DNA, green letters 2'F RNA, and blue letters cEt modifications. All oligonucleotides are PS-modified, except for underlined letters, which are natural phosphodiesters (PO). Oligos with a mixture of PS and PO linkages are mixed backbone (MBB); X = Alexa 647 Fluor. The number in parentheses is a value from duplicate measurements.

that the receptor has a significantly higher affinity for singlestranded ASO (ASO 1) than for double-stranded molecules in which the same ASO was bound with a RNA complement (ASO 8). The phosphorothioate linkage is highly preferred for binding (ASO 1) in contrast to the phosphodiester oligo (ASO 5 ), and the affinity rapidly drops as the oligo length is reduced to 15 bases (ASO 6) and 10 bases (ASO 7). The five flanking bases with $2^{\prime}$ modifications did not affect the overall affinity, indicating that the PS modification on the oligo backbone is the primary contributor for Stabilin-2 binding (compare ASO 5 with ASO 1, 9, and 10).

The same experiments were repeated using a "gapmer" designed set of oligos (Figure 2B and Table 2). The control for this group is PTEN ASO 1. All of these PS-based oligos (ASO 11-15) bound with affinities within 1 order of magnitude of each other (15-73 nM). The $\mathrm{T}_{20}$ (ASO 17) and $\mathrm{A}_{20}$ (ASO 18) oligos were also compared against each other, which resulted in $\mathrm{A}_{20}$ having a 20 -fold weaker affinity for the receptor. The explanation for this observation is the assumption that because of the helical self-stacking of the purine bases, $A_{20}$ is more rigid

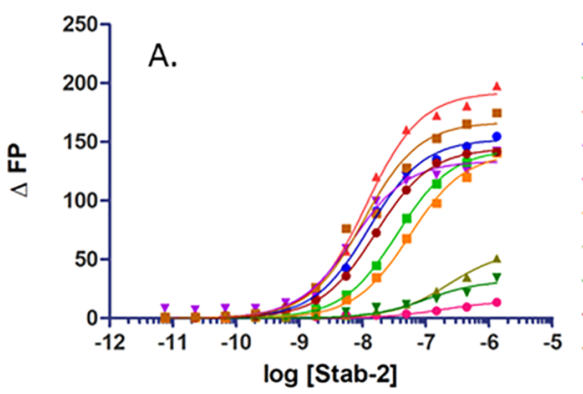

$\rightarrow$ ASO\# 1

- ASO\# 2

$\rightarrow$ ASO\# 3

$\rightarrow$ ASO\# 4

- ASO\# 5

- ASO\# 6

- ASO\# 7

$\rightarrow$ ASO\# 8

$\rightarrow$ ASO\# 9

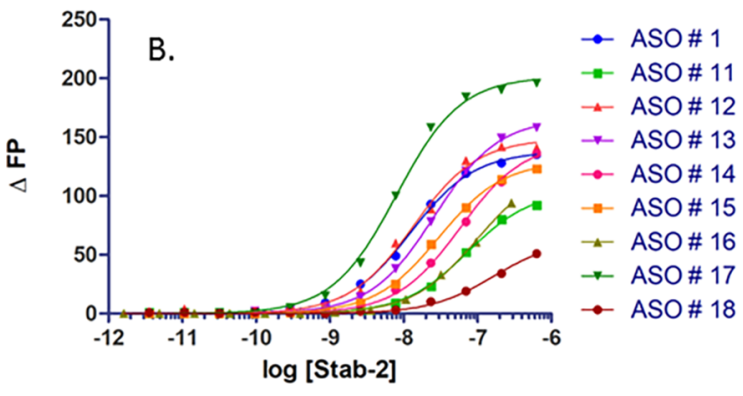

Figure 2. Direct ASO binding to s190 (Stab2). (A) PTEN ASOs with various chemistries. (B) ASOs with differing sequences and designs.

Table 2. Effect of Sequence and Design on Binding to s190 $\left(\right.$ Stab2) ${ }^{a}$

\begin{tabular}{|c|l|l|l|}
\hline ASO & Sequence & ASo Design & $\begin{array}{l}\text { Kd } \\
\text { (nM) }\end{array}$ \\
\hline $\mathbf{1}$ & X-CTGCTAGCCTCTGGATTTGA & PTEN 5-10-5 MOE PS & 12.4 \\
\hline $\mathbf{1 1}$ & X-GCTCCTTCCACTGATCCTGC & PTB1B 5-10-5 MOE PS & 73.4 \\
\hline $\mathbf{1 2}$ & X- TGCATCCCCCAGGCCACCAT & ICAM1 DNA PS & 9.0 \\
\hline $\mathbf{1 3}$ & X-TCCCATTTCAGGAGACCTGG & CRP 3-14-3 MOE PS & 26.2 \\
\hline $\mathbf{1 4}$ & X-GCTGATTAGAGAGAGGTCCC & TNF- $\alpha$ 5-10-5 MOE PS & 58.6 \\
\hline $\mathbf{1 5}$ & X-CCTTCCCTGAAGGTTCCTCC & Control 5-10-5 MOE PS & 29.8 \\
\hline $\mathbf{1 6}$ & X-NNNNNNNNNNNNNNNNNNNN & A-basic & 96.5 \\
\hline $\mathbf{1 7}$ & X-TTTTTTTTTTTTTTTTTTTT & T $_{20}$ DNA PS & 7.9 \\
\hline $\mathbf{1 8}$ & X-AAAAAAAAAAAAAAAAAAAA & A $20_{20}$ DNA PS & 165 \\
\hline
\end{tabular}

${ }^{a}$ Orange letters indicate MOE and black letters DNA. N indicates a PS-modified abasic oligonucleotide, and X = Alexa 647 Fluor.

and, therefore, has a lower binding affinity. Rigidity may also be the reason for which the double-stranded PTEN ASO has an affinity lower than that of the single-stranded ASO in Table 1. The tight binding with the A-basic ASO (ASO 16) confirms that the PS modification is the binding motif for Stabilin-2, which is in agreement with the PTEN ASOs in which the PO version of the PTEN ASO does not bind with s190.

The effect of $\mathrm{pH}$ on ASO binding is important to examine as once the receptor is internalized in early endosomes, the $\mathrm{pH}$ decreases during endosomal maturation. Using a mixture of mono- and divalent $10 \mathrm{mM}$ sodium phosphate buffers containing $150 \mathrm{mM} \mathrm{NaCl}$, the fluorescence-based assay was repeated under four $\mathrm{pH}$ conditions (Figure $3 \mathrm{~A}$ ). As the $\mathrm{pH}$ decreased from 7.4 to 5.0, the affinity decreased. For most protein-ASO interactions that have been observed thus far, the opposite trend or no change in binding affinity is the typical result. In other proteins and receptors, the higher affinity at lower $\mathrm{pH}$ may be the result of a more positive charge that is attractive to the polyanionic PS ASO. ${ }^{26}$ However, this is clearly not the case with this receptor. Most, if not all, professional endocytic receptors release their cargo under low- $\mathrm{pH}(<5.5)$ conditions, ${ }^{27}$ and this may be the reason for the results observed for Stabilin-2. 

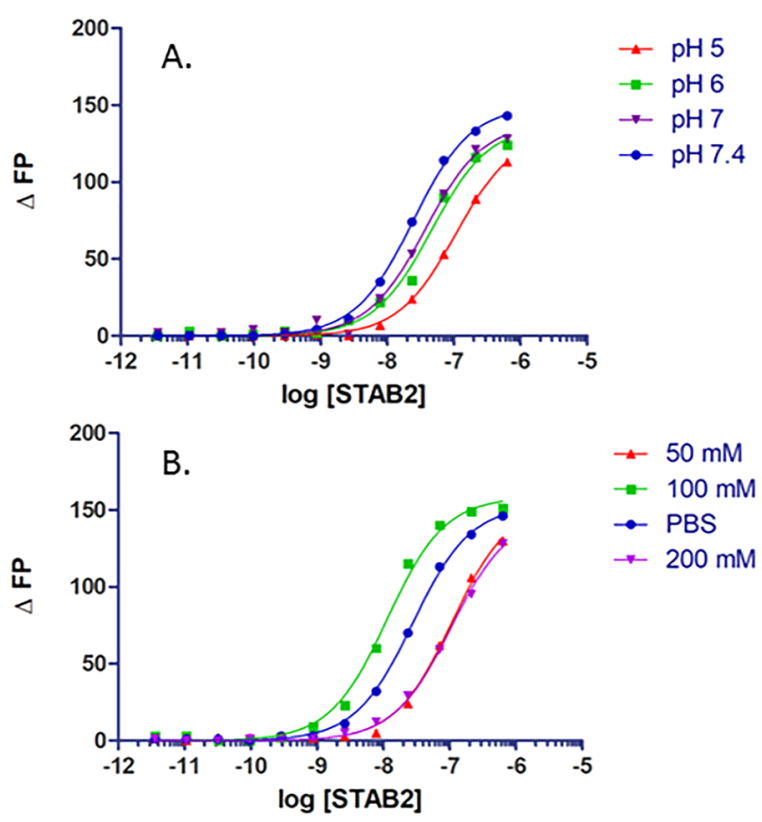

Figure 3. ASO 1 binding to $\mathrm{s} 190$ under various (A) $\mathrm{pH}$ and (B) salt conditions. The phosphate-buffered saline concentration is $150 \mathrm{mM}$.

Previous experiments with an ELISA type assay revealed that PS ASO-protein binding is dependent on ionic bonding. ${ }^{8} \mathrm{We}$ repeated this assay with the FP method and found that, as before, the affinity of the ASO for the receptor decreases as the ionic strength increases (Figure 3B). It is somewhat surprising that binding affinity is weakest at the lowest salt concentration and may be a result of an artifact from the experimental method or that low concentrations of salt perturb protein structure enough to decrease the level of ASO binding. Any perturbation to salt concentration may alter the physical and chemical environment of the binding site(s). There are no structural data for this receptor, and the s190 used in these experiments contains 1359 amino acids, including 104 cysteine residues; thus, predicting overall and specific subdomain structures is not possible at this time (Figure S1).

This fluorescence-based assay confirmed the results from our previous report describing the high affinity of the PS-based ASO for the Stabilin receptors. ${ }^{8}$ This is the first report in which direct binding affinities have been observed with a multitude of different PS and non-PS ASOs that could not have been attained otherwise. With this information, it is clear that the length of the PS backbone and the single-stranded nature of the nucleic acid are the primary determinants for binding to the Stabilin receptors. In addition, the nucleotide sequence does not substantially affect the affinity for the receptor.

The sequence-independent tissue accumulation properties of PS ASOs in the liver have been used advantageously for the clinical development of ASO therapeutics. Our binding data show that PS ASOs can bind the Stabilin receptors, and presumably other cell-surface proteins, in a PS-dependent but sequence-independent manner and provide a rationale for the predictable liver accumulation properties of single-stranded PS ASOs in animals. Our data also emphasize the importance of interactions with cell-surface proteins for the promotion of cellular internalization of nucleic acid-based therapeutics.

\section{ASSOCIATED CONTENT}

\section{Supporting Information}

The Supporting Information is available free of charge on the ACS Publications website at DOI: 10.1021/acs.biochem.8b00126.

Methodology for the purification of s190 and fluorescence polarization assay and the amino acid sequence and domain organization of s190 (Figure S1) (PDF)

\section{AUTHOR INFORMATION}

\section{Corresponding Authors}

*Department of Biochemistry, University of Nebraska, 1901 Vine St., Beadle N133, Lincoln, NE 68588. Telephone: 402472-7468. E-mail: eharris5@unl.edu.

*Medicinal Chemistry, Ionis Pharmaceuticals, 2855 Gazelle Ct., Carlsbad, CA 92010. Telephone: 760-603-2587. E-mail: pseth@ionisph.com.

ORCID $\odot$

Edward N. Harris: 0000-0001-7045-1040

Funding

The authors are grateful for funding from National Institutes of Health Grant R01HL130864 to E.N.H.

\section{Notes}

The authors declare no competing financial interest.

\section{ACKNOWLEDGMENTS}

The authors are grateful to Tracy Reigle (Ionis Pharm.) for her assistance with manuscript preparation.

\section{REFERENCES}

(1) Shen, X., and Corey, D. R. (2018) Nucleic Acids Res. 46, 15841600.

(2) White, P. J., Anastasopoulos, F., Pouton, C. W., and Boyd, B. J. (2009) Expert Rev. Mol. Med. 11, No. e10, DOI: 10.1017/ S1462399409001021.

(3) Crooke, S. T., Wang, S., Vickers, T. A., Shen, W., and Liang, X. H. (2017) Nat. Biotechnol. 35, 230-237.

(4) Eckstein, F. (2014) Nucleic Acid Ther. 24, 374-87.

(5) Geary, R. S., Norris, D., Yu, R., and Bennett, C. F. (2015) Adv. Drug Delivery Rev. 87, 46-51.

(6) Monia, B. P., Lesnik, E. A., Gonzalez, C., Lima, W. F., McGee, D., Guinosso, C. J., Kawasaki, A. M., Cook, P. D., and Freier, S. M. (1993) J. Biol. Chem. 268, 14514-22.

(7) Wan, W. B., and Seth, P. P. (2016) J. Med. Chem. 59, 9645-9667.

(8) Miller, C. M., Donner, A. J., Blank, E. E., Egger, A. W., Kellar, B. M., Ostergaard, M. E., Seth, P. P., and Harris, E. N. (2016) Nucleic Acids Res. 44, 2782-94.

(9) Politz, O., Gratchev, A., McCourt, P. A., Schledzewski, K., Guillot, P., Johansson, S., Svineng, G., Franke, P., Kannicht, C., Kzhyshkowska, J., Longati, P., Velten, F. W., Johansson, S., and Goerdt, S. (2002) Biochem. J. 362, 155-64.

(10) Kzhyshkowska, J., Gratchev, A., and Goerdt, S. (2006) J. Cell. Mol. Med. 10, 635-49.

(11) Weigel, J. A., and Weigel, P. H. (2003) J. Biol. Chem. 278, 42802-11.

(12) Falkowski, M., Schledzewski, K., Hansen, B., and Goerdt, S. (2003) Histochem. Cell Biol. 120, 361-9.

(13) Park, S. Y., Yun, Y., Lim, J. S., Kim, M. J., Kim, S. Y., Kim, J. E., and Kim, I. S. (2016) Nat. Commun. 7, 10871.

(14) Kyosseva, S. V., Harris, E. N., and Weigel, P. H. (2008) J. Biol. Chem. 283, 15047-55.

(15) Pempe, E. H., Xu, Y., Gopalakrishnan, S., Liu, J., and Harris, E. N. (2012) J. Biol. Chem. 287, 20774-83. 
(16) Park, S. Y., Jung, M. Y., Kim, H. J., Lee, S. J., Kim, S. Y., Lee, B. H., Kwon, T. H., Park, R. W., and Kim, I. S. (2008) Cell Death Differ. 15, 192-201.

(17) Park, S. Y., Jung, M. Y., Lee, S. J., Kang, K. B., Gratchev, A., Riabov, V., Kzhyshkowska, J., and Kim, I. S. (2009) J. Cell Sci. 122, 3365-73.

(18) Li, R., Oteiza, A., Sorensen, K. K., McCourt, P., Olsen, R., Smedsrod, B., and Svistounov, D. (2011) Am. J. Physiol Gastrointest Liver Physiol 300, G71-81.

(19) Kzhyshkowska, J., Workman, G., Cardo-Vila, M., Arap, W., Pasqualini, R., Gratchev, A., Krusell, L., Goerdt, S., and Sage, E. H. (2006) J. Immunol. 176, 5825-32.

(20) Kzhyshkowska, J., Gratchev, A., Schmuttermaier, C., Brundiers, H., Krusell, L., Mamidi, S., Zhang, J., Workman, G., Sage, E. H., Anderle, C., Sedlmayr, P., and Goerdt, S. (2008) J. Immunol. 180, 3028-37.

(21) Yannariello-Brown, J., Zhou, B., and Weigel, P. H. (1997) Glycobiology 7, 15-21.

(22) Harris, E. N., and Weigel, P. H. (2008) Glycobiology 18, 638-48.

(23) Harris, E. N., Kyosseva, S. V., Weigel, J. A., and Weigel, P. H. (2007) J. Biol. Chem. 282, 2785-97.

(24) Kornilova, A. Y., Algayer, B., Breslin, M., and Uebele, V. (2012) Anal. Biochem. 425, 43-6.

(25) Schmidt, K., Prakash, T. P., Donner, A. J., Kinberger, G. A., Gaus, H. J., Low, A., Ostergaard, M. E., Bell, M., Swayze, E. E., and Seth, P. P. (2017) Nucleic Acids Res. 45, 2294-2306.

(26) Vickers, T. A., and Crooke, S. T. (2016) PLoS One 11, No. e0161930.

(27) Niehrs, C., and Boutros, M. (2010) Sci. Signaling 3, pe26. 